\title{
Characterizing Kripke Structures in Temporal Logic
}

\author{
M. C. Browne \\ F. M. Clarke \\ O. Grümbcrg \\ Carnegic Mellon University, Pittsburgh
}

\section{Introduction}

The question of whether brancling-time temporal logic or lincar-time temporal logis is best for reasoning about concurrent programs is one of the most controyersial issues in logics of prograns. Concurrent programs are usually modelled by fabelled state-transition graphs in which some state is designated as the initial state. For historical reasons such graphs are called Kripke structures [8]. In lincar temporal logic, operators are provided for describing cvents along a single time path (i.e., along a single path in a Kripke structure). In a branching-time logic the temporal operators quantify over the futures that ale possible from a given state (i.e., over the possible paths that lead from a state). It is well known that the two types of temporal logic have different expressive powers ([4]. [9]). Lincar temporal logic, for example, can express certain fairness properties that cannot be expressed in branching-time temporal logic. On the other hand, certain practical decision problems like model checking ( $[3],[16]$ ) are easier for branching-time temporal logic than for linear temporal logic.

In this paper we provide further insight on which type of logic is best. We show that if two finite Kripke structures can be distinguished by some formula that contains both branching time and lincar-time operators, then the structurcs can be distinguished by' a formula that contains only branching time operators. Spccifically, we show that if two finite Kripke structures can be distinguished by some formula of the logic $\mathrm{CTL}^{*}$ (i.e., if there is some CTL ${ }^{*}$ formula that is true in one but not in the other), then they can be distinguished by some formula of the logic CTL. The logic CTL* ([3], [4]) is a very powerful temporal logic that combines both branching-time and linear-time opcrators; a path quantifier, either A ("for all path") or E ("for some paths") can prefix an assertion composed of arbitrary combinations of the ususal linear time operators G ("always"), F ("sometimes"), X ("nexttime"), and U ("until"). CTL ( [1], [2]) is a restricted subset of CTL" that permits only branching-time operators--each path quantifier must be immediately followed by exactly one of the operators $\mathrm{G}, \mathrm{F}, \mathrm{X}$, or $\mathrm{U}$.

Our goal is to show that for any finite Kripke structure $M$, it is possible to construct a CTL formula $F_{M}$ that uniquely characterizes $M$. Since one Kripke structure may be a trivial unrolling of another, we use a notion of equivalence between Kripke structures that is similar to the notion of bisimulation studied by Milner [12]. We say that states $s$ and $s^{\prime}$ are equivalent if they have the same labelling of atomic propositions and for each transition from one of the two states to some state $t$ there is a corresponding transition from the other state to a state $t^{\prime}$ that is equivalent to $t$. Two Kripke structures are equivalent if their initial states are equivalent. It is not difficult to prove that if two Kripke structures are equivalent, then their initial states must satisfy the same $\mathrm{CTL}^{*}$.

This research was parially supported by NSF Grant MCS-82-16706. The third author, O. Grümberg, is currently on leave from Technion, Haifa and is partially supported by a Weizmann postdoctoral fellowship. 
An obvious first attempt to construct $F_{M}$ is simply to write a C'I. formula that specifies the transition relation of $M$. For each state $\sin M /$ we include in $F_{M}$ a conjunct of the form

$$
\Lambda G\left(\mathcal{L}(s) \Rightarrow \bigwedge_{i} \mathrm{EX} \mathcal{L}\left(s_{i}\right) \wedge \Lambda \mathrm{X}\left(\vee_{i} \mathcal{L}\left(s_{i}\right)\right)\right)
$$

where $s_{1}, \ldots, s_{n}$ are the successors of $s$ and $\ell(t)$ is the labelling of atomic propositions associated with state $t$. It is casy to see, however, that this simple approach cannot work in general: several states in $M$ may have exactly the same labelling of atomic propositions.

Instead, we first show that it is possible to write a CTL formula that will distinguish between two states in the same structure that are not equivalent according to the above definition. Two incquivalent states may have exactly the same labelling of atomic propositions, they may even have corresponding successors, but the computation trees rooted at those states must differ at some finite depth. The difference in the computation trees can be exploited to give a CTL formula that distinguishes between the states. Since cquivalent states satisfy the same CTL" formulas, it follows that if two states can be distinguished by a CTL" formula, they can be distinguished by a ClL formula. Once we can distinguish between inequivalent states in the same structure, we can write a single CrL, formula that encodes the entire Kripke structure; this formula is the $F_{M}$ that we seek.

The above construction requires the use of the nexttime operator in specifying $F_{M}$. In reasining about concurrent systcms, however, the nexttime operator may be dangerous, since it refers to the global next state instead of the local next state within a process [10]. What happens if we disallow the nextime operator in CrL formulas? The proof, in this case, requires another notion of equivalence--equivalence with respect to shutlering. We say that two state sequences correspond if each can be partitioned into finite blocks of identically labelled states such that each state in the $i$-th block in one sequence is equivalent to each state in the $i$-th block of the other sequence. Thus, duplicating some state in a sequence any finite number of times will always result in a corresponding sequence. We say that two states are equivalent if for each state sequence starting at one there is a corresponding state sequence that starts at the other. Under this second notion of equivalence the proof of the characterization theorem becomes much more complicated, since it is possible for two inequivalent states to have exactly the same finite behaviors (modulo stuttering), but different infinite behaviors.

Equivalence under stuttering turns out to be quite useful for reasoning about hierarchically constructed concurrent systems. In determining the correctness of such a system by using a technique like temporal logic model checking ([2], [3], [11], [13], [16], [17]), it is often desirable to replace a low level module by an equivalent structure with fewer states. Our results show how this can be done while preserving all of those properties that are invariant under stuttering. We give polynomial algorithms both for determining if two structures are equivalent with respect to stuttering and for minimizing the number of states in a given structure under this notion of equivalence.

Finally, our results have some interesting implications for the problem of synthesizing finite state concurrent systems from temporal logic specifications ([2]. [14]). In order to guarantee that any Kripke structure can be synthesized from a specification in inear temporal logic, Wolper [18] was forced to introduce more complicated operators based on regular expressions. Our results show that (at least in theory) no such extension is necessary for branching-time temporal logic. Any Kripke structure can be specified directly by a formula of branching-time logic. 
The expressive power of various temporal logics has been discussed in several pupers; sec ([4], [9]) for example. Hennessy and Milner |7], Graf and Slakis [6], and Pnueli [15] have all discussed the relationship between temporal logic and various notions of equivalenec between models of concurrent prograns. However, we believe that we are the first to show that it is possible to characterize Kripke models within branching-time logic and to investigate the conscquences of this result.

Our paper is organized as follows: In Section 2 we describe the logics CTL and CTL". In Section 3, we state formally what it means for two states in a Kripke structure to be equivalent and prove that equivalent states satisfy exactly the same CCL." formulas. Section 3 also contains the first of the two main results of the paper: we show how to characterize Kripke structures using CTI formulas with the nexttime operator. Section 4 introduces the second notion of equivalence (equivalence with respect to stutlering) and shows that if the nexttime operator is disallowed, then equivalent states again satisfy exacty the same CTL" formulas. We also extend the characterization theorcm of Scetion 3 to Kripke structures with thy new notion of equivalence. In Section 5 we give a polynomial algorithm for detemining if two states are equivalent up to stuttering. The paper concludes in Section 6 with a discussion of some remaining open problems like the possibility of extending our results to Kripke structures with fairness constraints (i.e., Büchi Automata).

\section{The Logics CTL and CTL"}

There are two types of formulas in $\mathrm{CTL}^{*}$ : siate formulas (which are true in a specific state) and path formulas (which are true along a specific path). Let $A P$ be the set of atomic proposition names. $\Lambda$ state formula is either:

- $A$, if $A \in A P$.

- If $f$ and $g$ are state formulas, then $\neg f$ and $f \vee g$ are state formulas.

- If $f$ is a path formula, then $\mathbf{E}(f)$ is a state formula.

A path formula is either:

- A state formula.

- If $f$ and $g$ are path formulas, then $\neg f, f \vee g, X f$, and $f \mathbf{U} g$ are path formulas.

CTL ${ }^{*}$ is the set of state formulas generated by the above rules.

CTL is a subset of $\mathrm{CTL}^{*}$ in which we restrict the path formulas to be:

- If $f$ and $g$ are state formulas, then $\mathrm{X} f$ and $f \mathrm{U} g$ are path formulas.

- If $f$ is a path formula, then so is $\neg f$.

We define the semantics of both logics with respect to a structure $M=\langle S, R, \mathcal{L}\rangle$, where

- $S$ is a set of states.

- $R \subseteq S \times S$ is the transition relation, which must be total. We write $s_{1} \rightarrow s_{2}$ to indicate that $\left(s_{1}, s_{2}\right) \in R$.

- $2: S \rightarrow \mathscr{P}(A P)$ is the proposition labeling.

Unless otherwise stated, all of our results apply only to finite Kripke structures. 
We only consider transition relations where every state is reachable from the initial state. We define a path in $M$ to de a sequence of states, $\pi=s_{v}, s_{1}, \ldots$ such that for every $i \geq 0, s_{i} \rightarrow s_{i+1}, \pi^{i}$ will denote the suffix of $\pi$ starting at $s_{i}$.

We use the standard notation to indicate that a state formula $f$ holds in a structure: $M, s=f$ meatus that $f$ holds at state $s$ in structure $M$. Similarly, if $f$ is a path formula, $M, m \vDash f$ means that $f$ holds along path $\pi$ in structure $M$. The relation $F$ is defined inductively as follows (assuming that $f_{1}$ and $f_{2}$ are state formulas and $g_{1}$ and $g_{2}$ are path formulas):

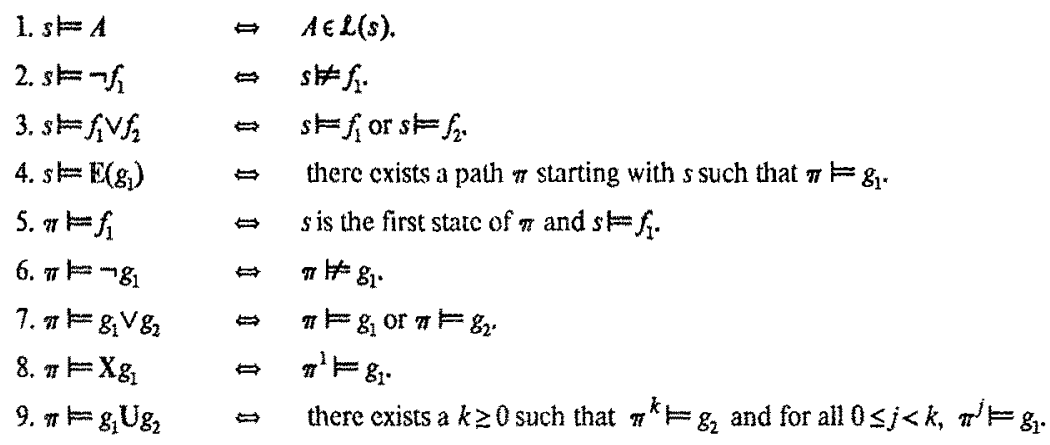

We will also use the following abbreviations in writing CTL" (and CTL) formulas:
$\cdot f \wedge g \equiv \neg(\neg f \wedge \neg g)$
- $\mathrm{F} f \equiv$ true U $f$
$\cdot \mathrm{A}(f) \equiv \neg \mathrm{E}(\neg f)$
$\bullet \mathrm{G} f \equiv \neg \mathrm{K} \neg f$.

\section{Equivalence of Kripke Structures}

Given two structures $M$ and $M^{\prime}$ with the same set of atomic propositions $A P$, we define a sequence of equivalence relations $E_{0}, E_{1}, \ldots$ on $S \times S^{\prime}$ as follows:

- $s E_{0} s^{\prime}$ if and only if $\ell(s)=L\left(s^{\prime}\right)$.

- $s E_{n+1} s^{\prime}$ if and only if

$$
\begin{aligned}
& \circ \mathcal{L}(s)=\mathcal{L}\left(s^{\prime}\right), \\
& \circ \forall s_{1}\left[s \rightarrow s_{1} \Rightarrow \exists s_{1}^{\prime}\left[s^{\prime} \rightarrow s_{1}^{\prime} \wedge s_{1} E_{n} s_{1}^{\prime}\right]\right], \text { and } \\
& \circ \forall s_{1}^{\prime}\left[s^{\prime} \rightarrow s_{1}^{\prime} \Rightarrow \exists s_{1}\left[s \rightarrow s_{1} \wedge s_{1} E_{n} s_{1}^{\prime}\right]\right]
\end{aligned}
$$

Now, we define our notion of equivalence berween states: $s E s^{\prime}$ if and only if $s E_{i} s^{\prime}$ for all $i \geq 0$. Furthermore, we say that $M$ with initial state $s_{0}$ is equivalent to $M^{\prime}$ with initial state $s_{0}^{\prime}$ iff $s_{0} E s_{0}^{\prime}$.

Lemma 1: Let $s E s^{\prime}$, then for every path, $s_{,}, \ldots$, there exists a path, $s^{\prime}, s_{1}^{\prime} \ldots$ such that $\forall i\left[s_{i} E s_{i}^{\prime}\right]$.

Proof: Note first that $E_{n+1} \subseteq E_{n}$. Since $E_{0}$ is finite, there must be a $k$ such that $E_{k+1}=E_{k}=E$. Thus, we can substitute $E$ for $E_{k}$ in the definition of $E_{k+1}$ giving $s E s^{\prime}$ if and only if

- $\mathcal{L}(s)=L\left(s^{\prime}\right)$, 
- $\left.\forall s_{j}\left[s \rightarrow s_{1} \Rightarrow \exists s_{1}^{\prime} \mid s^{\prime} \rightarrow s_{1}^{\prime} \wedge s_{1} E_{n} s_{l}^{\prime}\right]\right]$, and

- $\forall s_{j}^{\prime}\left[s^{s} \rightarrow s_{1}^{t}=\exists s\left[s \rightarrow s_{1} \wedge s_{1} E_{n} s_{1}^{t}\right]\right]$

The remainder of the proof is a straightforward induction on the length of the path.

Theorem 2: If $s E s^{\prime}$, then $\forall f \in C T L . "\left[s=f \Leftrightarrow s^{\prime}=f\right]$.

This theorem is a consequence of the following lemma:

Lermina 3: I.et $h$ be either a state formula or a path formula. Let $\pi=s_{1} s_{1}, \ldots$ be a path in $M$ and $\pi^{\prime}=s^{\prime}, s_{1}^{\prime}$. be a path in $M^{\prime}$ such that $s E s^{\prime}$ and $\forall i\left[s_{i} E s_{i}^{\prime}\right]$. Then

$s \equiv h \Leftrightarrow s^{\prime} \models h$, if $h$ is a state formula and

$\pi \vDash h \Leftrightarrow \pi^{\prime} \vDash h$, if $h$ is a path formula.

Proof: We prove the theorem by induction on the structure of $h$.

Base: $h=A$. By the definition of $E, s \neq A \Leftrightarrow s^{\prime} \vDash A$.

Induction: There are several cases.

1. $h=\neg h_{1}$, a state formula.

$$
\begin{aligned}
s \neq h & \Rightarrow s \neq h_{1} \\
& \Rightarrow s^{\prime} \neq h_{1} \text { (induction hypothesis) } \\
& \Rightarrow s^{\prime}=h
\end{aligned}
$$

The same reasoning holds if $h$ is a path formuia.

2. $h=h_{1} \vee h_{2}$, a state formula.

Without loss of generality,

$$
\begin{aligned}
s \neq h & \Leftrightarrow s \vDash h_{1} \text { or } s \vDash h_{2} \\
& =s \models h_{1} \\
& \Leftrightarrow s^{\prime} \vDash h_{1} \text { (induction hypothesis) } \\
& \Rightarrow s^{\prime} \vDash h
\end{aligned}
$$

The argument is the same in the other direction. We can also use this argument if $h$ is a path formula.

3. $h=\mathbf{E}\left(h_{1}\right)$, a state formula.

Suppose that $s=h$. Then there is a path, $\pi_{1}$ starting with $s$ such that $\pi_{1} \models h_{2}$. By Lemma 1 , there is a corresponding path $\pi_{1}^{\prime}$ in $M^{\prime}$ starting with $s^{\prime}$. So by the induction hypothesis, $\pi_{1} \vDash h_{1} \Leftrightarrow \pi_{1}^{\prime} \vDash h_{1}$. Therefore, $s \models \mathrm{E}\left(h_{1}\right) \Rightarrow s^{\prime} \models \mathrm{E}\left(h_{1}\right)$. We can use the same argument in the other direction, so the lemma holds.

4. $h=h_{1}$, where $h$ is a path formula and $h_{1}$ is a state formula. 
Although the lengths of $h$ and $h_{1}$ are the same, we can imagine that $h=$ path $\left(h_{1}\right)$, where path is an sperator which converts a state formula into a path formula. Therefore, we are simplifying $h$ by dropping this path operator. So now:

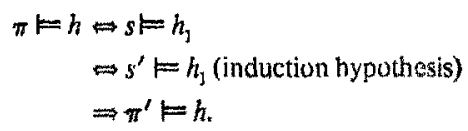

The reverse direction is similar.

5. $h=\mathrm{X} h_{1}$, a path formula.

By the definition of the next-time operator, $\pi^{1}=h_{1}$. Since $\pi$ and $\pi^{\prime}$ correspond, so do $\pi^{2}$ and $\pi^{\prime 1}$. Therefore, by the inductivc hypothesis, $\pi^{\prime \prime}=h_{1}$ so $\pi^{\prime}=h$.

We can use the same argument in the other direction.

6. $h=h_{1} \mathrm{U} h_{2}$, a path formula.

Suppose that $\pi \models h_{1} \mathrm{U} h_{2}$. By the definition of the until operator, there is a $k$ such that $\pi^{k}=h_{2}$ and for all $0 \leq j<k, \pi^{j} \models h_{1}$. Since $\pi$ and $\pi^{\prime}$ correspond, so do $\pi^{j}$ and $\pi^{j}$ for any $j$. Thercfore, by the inductive hypothesis, $\pi^{i k} \models h_{2}$ and $\pi^{\prime j} \models h_{1}$ for all $0 \leq j<k$. Therefore $\pi^{\prime} \models h$.

We can use the same argument in the other direction.

Another property of two equivalent states is that they both have corresponding computation trees. For every $s \in S$, $\operatorname{Tr}_{n}(s)$ is the computation tree of depth $n$ rooted at $s$. Formally, $\operatorname{Tr}_{0}(s)$ consists of a single node which has the same label as $s$. $\operatorname{Tr}_{n+1}(s)$ has as its root a node $m$ with the same label as $s$. If $s$ has successors $s_{1}, \ldots, s_{p}$ in the Kripke structure, then node $m$ will have subtrees $\operatorname{Tr}_{n}\left(s_{1}\right), \ldots, \operatorname{Tr}_{n}\left(s_{p}\right)$.

Two trees $\operatorname{Tr}_{n}(s)$ and $\operatorname{Tr}_{n}\left(s^{\prime}\right)$ correspond (denoted $\operatorname{Tr}_{n}(s) \equiv \operatorname{Tr}_{n}\left(s^{\prime}\right)$ ) if and only if both of their roots have the same label and for every subtree of depth $n-1$ of the root of one, it is possible to find a corresponding subtree of the root of the other.

Lemma 4: $s E_{n} s^{\prime}$ if and only if $\operatorname{Tr}_{j}(s) \equiv \operatorname{Tr}_{j}\left(s^{\prime}\right)$ for all $j \leq n$.

Lemma 5: Given a finite set of states $s_{1} \ldots, s_{n}$ there exists a $c$ such that if two states $s_{i}$ and $s_{j}$ are not $E$-equivalent then $\mathrm{Tt}_{c}\left(s_{i}\right)$ and $\mathrm{Tr}_{c}\left(s_{j}\right)$ will not correspond.

We will call the value of $c$ for $S$ the characteristic number of the structure.

We associate a CTL formula with a tree $\operatorname{Tr}_{n}(s)$ as follows:

- $\because\left[\operatorname{Tr}_{0}(s)\right]=\left(p_{1} \wedge \ldots \wedge p_{u}\right) \wedge\left(\neg q_{1} \wedge \ldots \neg q_{v}\right)$, where $\mathcal{L}(s)=\left\{p_{1}, \ldots, p_{u}\right\}$ and $A P-\mathcal{L}(s)=\left\{q_{1}, \ldots, q_{v}\right\}$. 


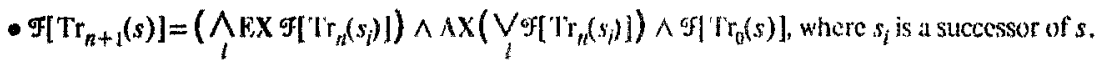

Lemma 6: $s \vDash \mathscr{F}\left[\operatorname{Tr}_{n}(s)\right]$ for all $n \geq 0$.

Lemma 7: If $s=\mathscr{F}\left[\operatorname{Tr}_{n}\left(s^{\prime}\right)\right]$, then $\operatorname{Tr}_{n}(s) \equiv \operatorname{Tr}_{n}\left(s^{\prime}\right)$.

Proof: The proof is by induction on $n$. The basis case is trivial. Thus, we assume that $n>0$. Let $s_{1}, s_{2}, \ldots, s_{p}$ be the sons of $s$ in $\operatorname{Tr}_{n}(s)$ and $s_{2}^{\prime}, s_{2}^{\prime}, \ldots, s_{q}^{\prime}$ be the sons of $s^{\prime}$ in $\operatorname{lr}_{n}\left(s^{\prime}\right)$.

It is easy to see that $s$ and $s^{\prime}$ have the same labelling of atomic propositions.

We must show that $\operatorname{Tr}_{n-1}\left(s_{i}\right)$ corresponds to some $\operatorname{Tr}_{n-1}\left(s_{j}^{\prime}\right)$. Since $s \equiv \mathscr{F}\left[\operatorname{Tr}_{n}\left(s^{\prime}\right)\right], s \neq \Lambda X\left(V_{j} F\left[\operatorname{Tr}_{n-1}\left(s_{j}^{\prime}\right)\right]\right)$. Since $s_{i_{0}}$ is a successor of $s, s_{i_{0}}=\sigma\left[\operatorname{Tr}_{n-1}\left(s_{j_{0}}^{\prime}\right)\right]$ for somc $j_{0}$. Hence, $\operatorname{Tr}_{n-1}\left(s_{i_{0}}\right) \equiv \operatorname{Tr}_{n-1}\left(s_{j_{9}^{\prime}}^{\prime}\right)$ by our inductivc hypothesis.

Finally, we must show that $\operatorname{Tr}_{n-1}\left(s_{j_{0}}^{\prime}\right)$ corresponds to some $\operatorname{Tr}_{n-1}\left(s_{i}\right)$. Since $\left.s=\mathscr{F}\left[\operatorname{Tr}_{n}\left(s^{\prime}\right)\right], s \neq \bigwedge_{j} \mathbb{E} \mathscr{X F}_{\left[r_{n-1}\right.}\left(s_{j}^{\prime}\right)\right]$. Since $s_{j_{0}}^{\prime}$ is a successor of $s^{\prime}, s=\operatorname{EXF}\left[\operatorname{Tr}_{n-1}\left(s_{j_{6}}^{\prime}\right)\right]$. Thercfore, there exists an $i_{0}$ such that $s_{i} \models g f\left[T_{n-1}\left(s_{j}^{\prime}\right)\right]$. Hence, $\operatorname{Tr}_{n-1}\left(s_{i_{0}}\right) \equiv \operatorname{Tr}_{n-1}\left(s_{j_{0}}^{\prime}\right)$ by our inductive hypothesis.

Lemma 8: If $s$ is a state in a Kripke structure $M$, then there is a CTL formula, $\mathrm{C}(M, s)$ that determines $s$ up to $E$-equivalence within $M$, i.c. $C(M, s)$ is true in $s$ and cvery state in $M$ that is $E$-equivalent to $s$ but false in every state in $M$ that is not equivalent to $s$.

Proof: We choose $\mathcal{C}(M, s)=\mathscr{G}\left[\mathrm{Tr}_{c}(s)\right]$ where $c$ is the characteristic number of $M . \mathcal{C}(M, s)$ is true in $s$ and hence in all states $E$-equivalent to $s$. Let $s^{\prime}$ be a state that is not $E$-equivalent to $s$; then $\operatorname{Tr}_{c}(s) \neq \operatorname{Tr}_{c}\left(s^{\prime}\right)$. Hence, by lemma 7 , $s^{\prime} \not \neq C(M, s)$.

Theorem 9: Given a Kripke structure $M$ with initial state $s_{0}$, there is a CTL formula $F\left(M, s_{0}\right)$ that characterizes that structure up to $E$-equivalence, i.e. $M^{\prime}, s_{0}^{\prime} \vDash F\left(M, s_{0}\right) \Leftrightarrow s_{0} E s_{0}^{\prime}$.

Proof: For any state $s$ in $M$, let $s_{1}, \ldots, s_{p}$ be the successors of $s$. We define

$$
G(M, s)=\mathrm{AG}\left(\mathrm{C}(M, s)=\bigwedge_{i} \mathrm{EX} C\left(M, s_{i}\right) \wedge \mathrm{AX} \underset{i}{\mathrm{C}}\left(M, s_{j}\right)\right)
$$

$G(M, s)$ describes all of the possible transitions from $s . F\left(M, s_{0}\right)$ is the formula $\mathcal{C}\left(M, s_{0}\right) \wedge \wedge G(M, s)$. If two structures $M, s_{0}$ and $M^{\prime}, s_{0}^{\prime}$ are equivalent then by theorem 2 they satisfy the same formulas. Since $M, s, s_{0}=F\left(M, s_{0}\right)$, so does $M^{\prime}, s_{0}^{\prime}$.

For the other direction we show by induction on $n$ that if $M^{\prime}, s_{0}^{\prime}=F\left(M, s_{0}\right)$ then $\operatorname{Tr}_{n}\left(s_{0}\right) \equiv \operatorname{Tr}_{n}\left(s_{0}^{\prime}\right)$ for all $n \geq 0$. By lemma 4 , the two structures are then $E$-equivalent.

Corollary 10: Given two structures $M$ and $M^{\prime}$ with initial states $s_{0}$ and $s_{0}^{\prime}$ respectively, $s_{0} E s_{0}^{\prime}$ if and only if $\forall f \in C T L^{*}\left[M, s_{0} \models f \Leftrightarrow M^{\prime}, s_{0}^{\prime} \vDash f\right]$.

Corollary 11: Given two structures $M$ and $M^{\prime}$ with initial states $s_{6}$ and $s_{0}^{\prime}$ respectively, if there is a formula of CTL" that is true in one and false in the other, then there is also a formula of CTL that is true in the one and false in the other. 
We illustrate our method of characterizing Kripke structures with the example in figure 3-1.

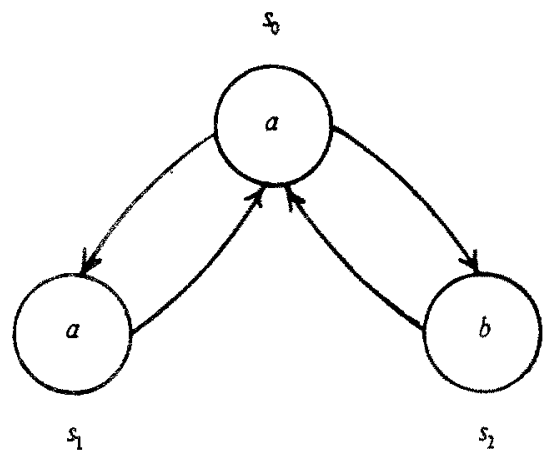

Figure 3-1: A Kripke structure in which every other state is labelled $A$

The characteristic number of this structure is 1 , since $\operatorname{Tr}_{0}\left(s_{0}\right) \neq \operatorname{Tr}_{0}\left(s_{2}\right), \operatorname{Tr}_{0}\left(s_{1}\right) \neq \operatorname{Tr}_{0}\left(s_{2}\right)$, and $\operatorname{Tr}_{1}\left(z_{0}\right) \neq \operatorname{Tr}_{1}\left(s_{1}\right)$. Let

- $C\left(M, s_{0}\right)=a \wedge \neg b \wedge \operatorname{EX}(a \wedge \neg b) \wedge \operatorname{EX}(\neg a \wedge b) \wedge \operatorname{AX}(a \wedge \neg b \vee \neg a \wedge b)$

- $C\left(M, s_{1}\right)=a \wedge \neg b \wedge \operatorname{EX}(a \wedge \neg b) \wedge \mathrm{AX}(a \wedge \neg b)$

- $\mathrm{C}\left(M, s_{2}\right)=\neg a \wedge b \wedge \operatorname{EX}(a \wedge \neg b) \wedge \mathrm{AX}(a \wedge \neg b)$

We can now state the formula that characterizes this structure:

$$
\begin{aligned}
F\left(M, s_{0}\right)= & \mathrm{C}\left(M, s_{0}\right) \wedge \\
& \operatorname{AG}\left(\mathrm{C}\left(M, s_{0}\right) \Rightarrow \operatorname{EXC}\left(M, s_{1}\right) \wedge \operatorname{EXC}\left(M, s_{2}\right) \wedge \operatorname{AXX}\left(C\left(M, s_{1}\right) \vee C\left(M, s_{2}\right)\right)\right) \wedge \\
& \operatorname{AG}\left(C\left(M, s_{1}\right) \Rightarrow \operatorname{EXC}\left(M, s_{0}\right) \wedge \operatorname{AXC}\left(M, s_{0}\right)\right) \wedge \\
& \operatorname{AG}\left(C\left(M, s_{2}\right) \Rightarrow \operatorname{EXC}\left(M, s_{0}\right) \wedge \operatorname{AXC}\left(M, s_{0}\right)\right)
\end{aligned}
$$

\section{Equivalence With Respect To Stuttering}

We first define what it means for two Kripke structures to be equivalent with respect to stuttering. Given two structures $M$ and $M^{\prime}$ with the same set of atomic propositions, we define a sequence of equivalence relations $E_{0}, E_{1}, \ldots$ on $S \times S^{\prime}$ as follows:

- $s E_{0} s^{\prime}$ if and only if $\mathcal{L}(s)=\mathcal{L}\left(s^{\prime}\right)$.

- $s E_{n+1} s^{\prime}$ if and only if

1. for every path $\pi$ in $M$ that starts in $s$ there is a path $\pi^{\prime}$ in $M^{\prime}$ that starts in $s^{\prime}$, a partition $B_{1} B_{2} \ldots$ of $\pi$, and a partition $B_{1}^{\prime} B_{2}^{\prime} \ldots$ of $\pi^{\prime}$ such that for all $j \in \mathbb{N}, B_{j}$ and $B_{j}^{\prime}$ are both non-empty and finite, and every state in $B_{j}$ is $E_{n}$-related to every state in $B_{j}^{\prime}$, and

2. for every path $\pi^{\prime}$ in $M^{\prime}$ starting in $s^{\prime}$ there is a path $\pi$ in $M$ starting in $s$ that satisfies the same condition as in 1.

Our notion of equivalence with respect to stutiering is defined as follows: $s E s^{\prime}$ if and only if $s E_{i} s^{\prime}$ for all $i \geq 0$. Furthermore, we say that $M$ with initial state $s_{0}$ is equivalent to $M^{\prime}$ with initial state $s_{0}^{\prime}$ if $s_{0} E s_{0}^{\prime}$. 
l, whuma 12: Given two Kripke stnetures $M$ and $M^{f}$, there cxiscs an / such that $\forall s \forall s^{\prime}\left[s E, s^{\prime}\right.$ iff $s E s^{\prime}$ ].

Proof: By the definition of $E_{l_{+}}, s F_{l+1}^{\prime} s^{\prime} \Rightarrow s E_{l} s^{\prime}$, so $E_{0} \supseteq E_{1} \supseteq E_{2} \ldots$ Since $M$ and $M^{\prime}$ are both finite, $F_{0}$ must be finite as well, so only a finite number of these containments can be proper. 1 et $E_{l}$ be the last relation that is properly included in $E_{l-1}$. By the definition of proper containment, $\forall m \geq l\left[E_{l}=E_{m}\right]$, so $s E_{l} s^{\prime} \Rightarrow s E_{m} s^{\prime}$, for $m \geq l$. Since $s E_{l} s^{\prime} \Rightarrow s E_{l-1} s^{\prime} \Rightarrow s E_{l-2} s^{\prime} \ldots$ we have $s E_{l} s^{\prime} \Rightarrow \forall m\left[s E_{m} s^{\prime}\right]$, so $s E_{l} s^{\prime} \Rightarrow s E s^{\prime}$. The other direction is trivial.

Theorem 13: If $s E s^{\prime}$, then for every CTL formula $f$ without the nextime operator, $s=f$ iff $s^{\prime} \vDash f$.

The proof is similar to that of theorem 2 .

lemma 14: Given a Kripke structure $M$, for every state $s \in M$, there is a CrL formula $C(M, s)$ such that $\forall t \in M[t \vDash C(M, s)$ iff $s E t]$.

Proof: We will prove by induction on l:

- If $\neg\left(s E_{l} l\right)$, then there is a CIL formula $d_{l}(s, i)$ such that $\forall v \in M\left[s E_{l} \nu \Rightarrow v \vDash d_{l}(s, l)\right]$ and $t \neq d(s, t)$.

- For every state $s \in M$, there is a CTL formula $C_{l}(M, s)$ such that for every $t \in M, t \models C_{l}(M, s)$ iff $s E_{l} t$.

$d_{l}(s, t)$ is a formula that distinguishes between $t$ and states equivalent to $s$ within the structure $M$, and $C_{l}(M, s)$ is a formula that characterizes $E_{l}$-equivalence to state $s$ within $M$.

If we let $\mathrm{C}_{l}(M, s)$ be a conjunction of $\mathrm{C}_{f-1}(M, s)$ and $d_{l}(s, t)$ for every $t$ that is not $E_{l}$-related to $s$ the second assertion follows easily. By lemma 12, this condition implies that the lemma is true. Now it is necessary to show how to construct $d_{l}(s, t)$ by induction on $l$.

Basis $(l=0)$ : Let $\left\{p_{i}\right\}$ be the set of atomic propositions in $\mathcal{L}(s)$ and $\left\{q_{j}\right\}$ be the set of atomic propositions in $A P-L(s)$. Now, let

$$
c_{0}(M, s)=d_{0}(s, t)=\bigwedge_{i} p_{i} \wedge \bigwedge_{j} \neg q_{j}
$$

It is clear that this formula is only true in states with the same labelling of atomic propositions as $s$. Therefore, the base case is established.

Induction: Assume that the result is true for $l$. We will show it for $l+1$.

Since $\neg\left(s E_{l+1} t\right)$, either there is a path from $s$ without a corresponding path from $t$, or vice versa. In the latter case, we will use the argument below to find a $d_{l+1}(t, s)$ such that $t \neq d_{l+1}(t, s)$ and $s \neq d_{l+1}(t, s)$. We can negate this formula to get the desired $d_{i+1}(s, t)$.

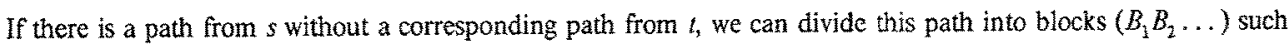
that:

$$
\forall i\left[x \in B_{i} \Rightarrow x \models \mathcal{C}_{l}\left(M, \text { first }\left(B_{i}\right)\right) \text { and first }\left(B_{i+1}\right) \neq \mathrm{C}_{l}\left(M, \text { first }\left(B_{i}\right)\right)\right] \text {. }
$$


Now, there are two cases: either there is a linite path fom one state without a corresponding path from the other, or there is an infinite path without a corresponding path, but cvery finite prefix of this path has a corresponding path.

In the first case, the path from sis finite, so the bloeks are finite and there are only a finite number of them (say n). Consider the $C T$. formula:

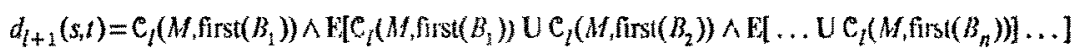

It is clear that $s=d_{l+1}(s, t)$ along the pati $B_{1} B_{2} \ldots B_{n}$. However, if $t=d_{t+1}(s . t)$ then there is a path that can be partitioned into blocks $B_{1}^{\prime} B_{2}^{\prime} \ldots B_{n}^{\prime}$ such that $\forall_{i}\left[v \in B_{i}^{\prime} \Rightarrow \eta \neq \mathcal{C}_{l}\left(M /\right.\right.$ first $\left.\left.\left(B_{i}\right)\right)\right]$. Since every state in $B_{i}$ satisfies $C_{l}\left(M, f i r s t\left(B_{i}\right)\right)$, the inductive hypothesis and the definition of $E_{l}$ gives $B_{i} E_{l} B_{j}^{\prime}$. Therefore, this path from $t$ corresponds to the path from $s$, a contradiction. We conclude that $/ \neq d_{l+1}(s, l)$.

In the second case, we start by showing that the path from $s$ has only a finite number of blocks by using an argument based on König's lemma. We can construct a tree rooted at / such that $t t_{1} \ldots t_{n}$ is a path through the tree if and only if therc is a path in the Kripke structurc $u_{1} \ldots u_{p} t_{1} v_{1} \ldots v_{q} t_{2} \ldots t_{n}$ that corresponds to a prefix of the path from $s$ with $B_{1}^{\prime}=\left\langle t u_{1} \ldots u_{p}\right\rangle, B_{2}^{\prime}=\left\langle t_{1} v_{1} \ldots v_{q}\right\rangle$, and so on. Now, if the path from $s$ has an infinite number of blocks, this tree must bave an infinite number of nodes. Otherwise, if the tree had $n$ nodes, there could be no path of length $n+1$, so the first $n+1$ blocks of the path from $s$ would have no corresponding path from $t$. Since the Kripke structure is finite, we also know that this tree must be finitcly branching. Thercfore, by König's lemma, there must be an infinite path through the tree. But this implies that there is an infinite path from $t$ that can be divided into an infinite number of blocks that correspond to the blocks of the path from $s$, so there is a path from $t$ corresponding to the path from $s$, violating our assumption. Therefore. the path from $s$ has only a finite number of blocks.

So, suppose that there are $n$ blocks, all of which are finite except the last. Consider the CTL formula:

$$
\left.d_{l+1}(s, t)=C_{l}\left(M, \text { first }\left(B_{1}\right)\right) \wedge \mathrm{E}_{[} \mathrm{C}_{l}\left(M_{\mathrm{f}} \mathrm{first}\left(B_{1}\right)\right) \cup \mathcal{C}_{l}\left(M, \text { first }\left(B_{2}\right)\right) \wedge \mathrm{E}\left[\ldots \mathrm{UEG} \mathrm{C}_{l}\left(M, \text { first }\left(B_{n}\right)\right)\right] \ldots\right]
$$

It is clear that $s=d_{i+1}(s, t)$ along the path $B_{1} B_{2} \ldots B_{n}$. However, if $t=d_{i+1}(s, t)$ then there is a path that can be partitioned into blocks $B_{1}^{\prime} B_{2}^{\prime} \ldots B_{n}^{\prime}$ such that all of the blocks are finite except $B_{n}^{\prime}$ and $\forall i\left[v \in B_{i}^{\prime} \Rightarrow \nu \models C_{l}\left(M\right.\right.$, first $\left.\left.\left(B_{i}\right)\right)\right]$. Since every state in $B_{i}$ satisfies $\mathcal{C}_{l}\left(M\right.$, first $\left.\left(B_{i}\right)\right)$, the inductive hypothesis and the definition of $E_{t}$ gives $B_{i} E_{l} B_{i}^{\prime}$. We can also divide the infinite blocks $B_{n}$ and $B_{n}^{\prime}$ into an infinite set of blocks containing one state each. Therefore, this path from 1 corresponds to the path from $s$, so we have a contradiction. We conclude that $t d_{l+1}(s, t)$.

Now, these $d_{l+1}(s, t)$ describe the existence or noncxistence of a single path along which some $C_{l}$ formulas hold. By the definition of $s E_{l+1} v$, every path from $s$ has a corresponding path from $v$ along which the same $\mathfrak{C}_{l}$ formulas hold and vice versa. Therefore, $s E_{l+1} v \Rightarrow v \neq d_{l+1}(s, t)$.

Therefore, the lemma is true.

Theorem 15: Given a Kripke structure $M$ with initial state $s_{0}$, there is a CTL formula $F\left(M_{2} s_{0}\right)$ that characterizes that structure up to $E$-equivalence with respect to stuttering, i.e. $M^{\prime}, s_{0}^{\prime} \vDash F\left(M, s_{0}\right) \Rightarrow s_{0} E s_{0}^{\prime}$.

Proof: For any state $s$ in $M$, let $s_{1}, \ldots, s_{p}$ be the extended successors of $s$, where an extended successor is a state that 
is not firelated to $s$ and is reachable from $s$ along a path consisting catirely of states that are liteguivalent to $s$. Next, we construct $G(M, s)$, which describes all of the transitions from sin $M$. In this construction, it is convenient to use the wak until operator, $A[f \mathrm{Wg}=-\mathrm{H}[\neg g \mathrm{U} \neg f \wedge \neg g]$, which differs from the ordinary until in that it permits an infinite path along which every state satisfies the first argument. So now:

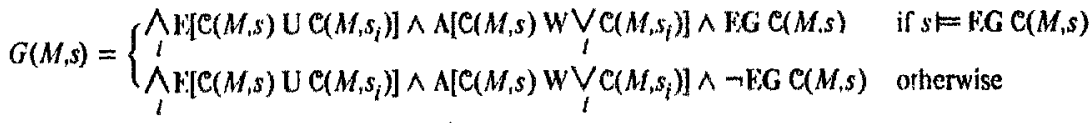

Let $F\left(M, s_{0}\right)$ be the formula $\mathrm{C}\left(M, s_{0}\right) \wedge \bigwedge_{s} \mathrm{AG}(\mathrm{C}(M, s) \Rightarrow G(M, s))$.

The correctness of $F\left(M, s_{0}\right)$ is an easy consequence of the next two lemmas and theorem 13 .

Lemma 16: $s=F(M, s)$.

Lemma 17: If $s=F(M, t)$ and $s^{\prime}=F(M, t)$, then $s E s^{\prime}$.

Proof of Lemma 16: Since every state is trivially equivalent to itself, $s=\mathcal{C}(M, s)$ is true by lemma 14. Therefore, if $s \not F(M, s)$ then there is a $t \in M$ such that $s=\operatorname{EF}(\mathbb{C}(M, l) \wedge \neg G(M, t))$. Let $v$ be the state reachable from $s$ that satisfies $\mathcal{C}(M, t) \wedge \neg G(M, t)$. By lemma 14 , this condition implies $t E v$, so $t$ and $v$ must satisfy the same CTL formulas (theorem 13). We will show that $t \neq \neg(M, l)$, giving a contradiction. There are four cases.

1. $t \equiv \mathrm{E}[\mathrm{C}(M, t) \cup \mathrm{C}(M, w)]$, for some extended successor of $t, w$. By the definition of extendcd successor, there is a path from $t$ to $w$ and the states on this path are $E$-related to $t$. By lemma 14 , these states must satisfy $C(M, t)$. Since $w \models C(M, w)$ is trivial, this path satisfics $C(M, t) \cup \mathcal{C}(M, w)$, which is a contradiction.

2. $t \neq \mathrm{EG} C(M, t)$. Since $\mathrm{EGC}(M, t)$ is a conjunct of $G(M, t)$ if and only if $t=\mathrm{EG} C(M, t)$, we have an immediate contradiction.

3. $t \neq-\mathrm{EG} \mathcal{C}(M, t)$. Since $\mathrm{EG} \neg \mathcal{C}(M, t)$ is a conjunct of $G(M, t)$ if and only if $t \neq \mathrm{EG} \mathcal{C}(M, t)$, we have an immediate contradiction.

4. $t \neq \mathrm{A}\left[\mathrm{C}(M, t) \mathrm{W} \bigvee_{i} \mathrm{C}\left(M, w_{i}\right)\right]$. In this case, $t=\mathrm{E}\left[(M, t) \cup\left(\neg \mathrm{C}(M, t) \wedge \wedge_{i} \neg \mathrm{C}\left(M, w_{i}\right)\right)\right]$. Let $t_{1} \ldots t_{n}$ be this path, where $t_{n} \Leftarrow \rightarrow \mathcal{C}(M, t) \wedge \wedge_{i} \neg \mathcal{C}\left(M, w_{i}\right)$ and $\forall i<n\left[l_{i} \models \mathcal{C}(M, t)\right]{ }^{i}$ By lemma $14, \neg\left(t_{n} E t\right)$ and

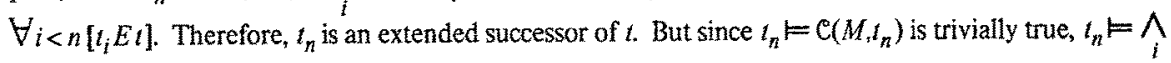
$\neg C\left(M, w_{i}\right)$ cannot be true, so we have a contradiction.

Therefore, the lemma is true.

Proof of Lemma 17: Since $s E s^{\prime}$ if and only if $s E_{l} s^{\prime}$ for all $l \geq 0$, we will prove $s \models F(M, t)$ and $s^{\prime} \vDash F(M, l)$ implies $s E_{l} s^{\prime}$ by induction on $l$.

Basis $(l=0)$ : Since $s \neq F(M, t) ; \quad s \neq C(M, l)$ and therefore $s=\mathrm{C}_{0}(M, t)$. Similarly, $s^{\prime} \neq \mathrm{C}_{0}(M, t)$, so $\mathcal{L}(s)=\mathcal{L}(l)=\mathcal{L}\left(s^{\prime}\right)$. Therefore, $s E_{0} s^{\prime}$. 
Induction: Assume that the result is true for $/$. We will now show it for $l+1$.

We want to show that every path, $\pi$. from $s$ has a corresponding path, $\pi^{\prime}$ from $s^{\prime}$. (The profof of the dual is identical.) We will use induction on the length of $\pi$ to prove the slightly stronger result:

If $|\pi| \leq n$, then there is a corresponding path $\pi^{\prime}$ such that for some $v \in M$, last $(\pi) \vDash F(M, v)$ and last $\left(\pi^{\prime}\right) \models F(M, v)$.

Basis $(|\pi|=1)$ : In this case, $\pi=\langle s\rangle$. Let $B_{1}=\langle s\rangle$ and $\pi^{\prime}=B_{1}^{\prime}=\left\langle s^{\prime}\right\rangle$. By the outer inductive hypothesis, $s \models F(M, t)$ and $s^{\prime} \models F(M, l)$ imply $s E_{l^{\prime}} s^{\prime}$, so $B_{1} E_{l} B_{1}^{\prime}$. 'Therefore, the paths correspond. Since the last states of each path satisfy $F(M, t)$, the base case is true.

Induction: Assume the result for $|\pi| \leq n$. Suppose that $\pi=s s_{1} s_{2} \ldots s_{n}$, a path of length $n+1$. Now, $s s_{1} s_{2} \ldots s_{n-1}$ is a path of length $n$, so by the inner inductive hypothesis, there is a corresponding path $\pi^{\prime}$ such that last $\left(\pi^{\prime}\right) \models F(M, v)$ and $s_{n-1} F F(M, v)$ for some $v \in M$. Let $B_{1} B_{2} \ldots B_{m}$ and $B_{1}^{\prime} B_{2}^{\prime} \ldots B_{m}^{\prime}$ be the partitions that show that these paths correspond. There are three cases.

1. $s_{n} k=\mathrm{C}(M, v)$. Since $s_{n-1} \models F(M, v)$, we can infer that $s_{n-1} \models \Lambda\left[C(M, v) W \underset{i}{\mathrm{C}}\left(M, w_{i}\right)\right]$. where $w_{i}$ are the cxtended successors of $v$. Since $s_{n-1} s_{n}$ is a path and $s_{n}$ that doesn't satisfy $C(M, v)$, we conclude that there must be an extended successor of $y, x$, such that $s_{n}=\mathcal{C}(M, x)$. Since $s_{n}$ is a successor of $s_{n-1}$, it must satisfy all of the AG formulas that $s_{n-1}$ satisfies, so $s_{n} \vDash F(M, x)$.

From last $\left(\pi^{\prime}\right) \models F(M, v)$ we can infer that last $\left(\pi^{\prime}\right) \models \mathfrak{C}(M, v) \wedge \mathrm{E}[\mathcal{C}(M, v) \cup \mathcal{C}(M, x)]$. Therefore, there is a path $s_{1}^{\prime} s_{2}^{\prime} \ldots s_{k}^{\prime}$ where $s_{1}^{\prime}=\operatorname{last}\left(\pi^{\prime}\right), \quad \forall i<k\left[s_{i}^{\prime} \models \mathcal{C}(M, v)\right]$, and $s_{k}^{\prime}=\mathcal{C}(M, x)$. Now let $\pi=B_{1} \ldots B_{m}\left\langle s_{n}\right\rangle$ and $\left.\pi^{\prime}=B_{1}^{\prime} \ldots B_{m-1}^{\prime}\left\langle B_{m}^{\prime}, s_{2}^{\prime} \ldots s_{k-1}^{\prime}\right\rangle s_{k}^{\prime}\right\rangle$. Since $s_{n}$ and $s_{k}^{\prime}$ both satisfy $F(M, x)$, the outer induction hypothesis gives $\left\langle s_{n}\right\rangle E_{l}\left\langle s_{k}^{\prime}\right\rangle$. Similarly, since the all the states in $B_{m} B_{m}^{\prime}$, and $\left\langle s_{2}^{\prime} \ldots s_{k-1}^{\prime}\right\rangle$ satisfy $F(M, v)$, they are all $E_{l}$ related to each other. Therefore, $\pi$ and $\pi^{\prime}$ correspond with last $(\pi) \vDash F(M, x)$ and last $\left(\pi^{\prime}\right) \vDash F(M, x)$.

2. $s_{n} \vDash C(M, v)$ and $v \models E G C(M, v)$. Since $s_{n}$ must satisfy the same AG formulas as $s_{n-1}, s_{n} \vDash F(M, v)$. Now, last $\left(\pi^{\prime}\right) \models F(M, v)$, so last $\left(\pi^{\prime}\right) \models \operatorname{EGC}(M, v)$. Therefore, last( $\left(\pi^{\prime}\right)$ must have a successor, $s_{1}^{\prime}$, which also satisfies $C(M, y)$. Since this state must also satisfy all of the AG formulas, $s_{1}^{\prime} \vDash F(M, v)$. Therefore, by the outer induction hypothesis, $s_{n} E_{l} s_{1}^{\prime}$. So if we let $B_{m+1}=\left\langle s_{n}\right\rangle$ and $B_{m+1}^{\prime}=\left\langle s_{1}^{\prime}\right\rangle$, the paths correspond.

3. $s_{n}=\mathcal{C}(M, v)$ and $v \neq \mathbf{E G} \mathcal{C}(M, v)$. By the reasoning above, $s_{n}=F(M, v)$, so $s_{n} E_{l}$ last $\left(B_{m}^{\prime}\right)$. Therefore, $\pi$ corresponds to $\pi^{\prime}$ with the same partition except that $s_{n}$ is added to $B_{m}$.

We must also show that the blocks of the partitions are finite. The only problem is case 3 , in which we might add an infinite number of states to a block of $\pi$. In this case, each of the states added to $B_{m}$ satisfy $F(M, v)$, so if we add an infinite number of states to this block first $\left(B_{m}\right)=\operatorname{EGC}(M, v)$ must be true. But since first $\left(B_{m}\right) \neq F(M, v)$, first $\left(B_{m}\right) \models \neg \operatorname{EGC}(M, v)$, so we have a contradiction. Therefore, all of the blocks of the partition must be finite.

Therefore, the lemma is true.

Corollary 18: Given two structures $M$ and $M^{\prime}$ with initial states $s_{0}$ and $s_{0}^{\prime}$ respectively, $s_{0} E s_{0}^{\prime}$ if and only if for all $\mathrm{CTL}^{*}$ formulas $f$ without the nexttime operator, $M, s_{0} \models f \Leftrightarrow M^{\prime}, s_{0}^{\prime} \vDash f$. 
Corollary 19: Given two structures, $M$ and $\boldsymbol{H}^{\prime}$ with initial states sond $s_{0}^{\prime}$ respectively, if there is a formula of $\mathrm{Cln}^{*}$ " without the nextime operator that is true in one and false in the other, then there is also a formula of Cll, without the nextlime operator that is true in the one and false in the other.

\section{Algorithm For Stuttering Equivalence}

In this Section we show how to compute the relation for equivalence with respect to stuttering for states within a single Kripke Structure $M$. The method that we suggest is polynomial in the number of states of $M$. To determine equivalence between states in two different Kripke structures $M_{1}$ and $M_{2}$, we form a Kripke structure $M_{12}$ that is the disjoint union of these structures and check equivalenee between the corresponding states in the combined structure.

We construct a relation $C$ on $S^{\prime} \times S$ that is identical to the relation $E$ defined in Section 4. $C=\bigcap_{n} C_{n}$ where $C_{n}$ is defined as follows:

$$
\text { - } C_{0}=\left\{\left(s, s^{\prime}\right) \mid \mathcal{L}(s)=\mathcal{L}\left(s^{\prime}\right)\right\}
$$

- In order to define $C_{n+1}$ we must first define the set $\operatorname{NEXT}_{n+1}(s)$ of extended successors of $s$. We define this set in terms of the set $\mathrm{ST}_{n+1}(s)$ of stuttering states of $s . \mathrm{ST}_{n+1}^{n+1}(s)=\bigcup_{k} \mathrm{ST}_{n+1}^{k}(s)$ where,

$$
\begin{aligned}
\circ \mathrm{ST}_{n+1}^{\circ}(s) & =\{s\} \\
\circ \mathrm{ST}_{n+1}^{k+1}(s) & =\mathrm{ST}_{n+1}^{k}(s) \cup\left\{s^{\prime} \mid s^{\prime} \notin \mathrm{ST}_{n+1}^{k}(s) \wedge \exists s^{\prime \prime} \in \mathrm{ST}_{n+1}^{k}(s)\left[s^{\prime \prime} \rightarrow s^{\prime}\right] \wedge s^{\prime} C_{n} s\right\} \\
\operatorname{NEXT}_{n+1}(s) & =\left\{s^{\prime} \mid s^{\prime} \notin \mathrm{ST}_{n+1}(s) \wedge \exists s^{\prime \prime} \in \mathrm{ST}_{n+1}(s)\left[s^{\prime \prime} \rightarrow s^{\prime}\right]\right\} .
\end{aligned}
$$

We will aiso use a predicate $\operatorname{LOOP}_{n}(s)$ that is true iff there is a cycie containing only states in $\mathrm{ST}_{n}(s)$.

Now we can define $C_{n+1}$ as follows:

$$
\begin{aligned}
C_{n+1}=\left\{\left(s, s^{\prime}\right) \mid\right. & \operatorname{LOOP}_{n+1}(s)=\operatorname{LOOP}_{n+1}\left(s^{\prime}\right) \wedge s C_{n} s^{\prime} \wedge \\
& \forall s_{1} \in \operatorname{NEXT}_{n+1}(s) \exists s_{1}^{\prime} \in \mathrm{NEXT}_{n+1}\left(s^{\prime}\right)\left[s_{1} C_{n} s_{1}^{\prime}\right] \wedge \\
& \forall s_{1}^{\prime} \in \mathrm{NEXT}_{n+1}\left(s^{\prime}\right) \exists s_{1} \in \mathrm{NEXT}_{n+1}(s)\left[s_{1} C_{n} s_{1}^{\prime}\right]
\end{aligned}
$$

Proof that the relation $C$ constructed above is actually equal to the relation $E$ defined in Section 4 will be given in the journal version of this paper. Since the inductive structures of the definitions of the two relations are different, it is necessary to split the proof into two parts: the first part shows that $E \subseteq C_{i}$ for every $i$; the second part shows that $C \subseteq E_{i}$ for every $i$.

Computing $\mathrm{ST}_{n}$ requires time $\mathrm{O}\left(|S|^{2}\right)$. Computing $C_{n+1}$ given $C_{n}$ requires time $\mathrm{O}\left(|S|^{4}\right)$, since at most $|S|^{2}$ pairs of states must be checked and each pair requires $O\left(|S|^{2}\right)$ time to check. The algorithm terminates as soon as $C_{n}=C_{n+1}$. Since at any previous step $k,\left|C_{k+1}\right|<\left|C_{k}\right|$ and since $C_{0}$ has at most $|S|^{2}$ pairs of states, there are at most $|S|^{2}$ steps in the construction of $C$. It follows that the complexity of the entire algorithm is $\mathrm{O}\left(|S|^{6}\right)$.

If we replace each equivalence class of $C$ by a single state, this algorithm can also be used to minimize the number of states in the structure. 


\section{Conclusion}

The results of our paper have a number of sumprising implications. For examplc. if a specification of a finite state concurrent program in $\mathrm{CTL}^{*}$ is sufficiently detailed so that there is snly one program (modulo one of our notions of equivalenee) that meets the specification, then an equivalent specification could have been writicn in CII. instead. Anuther surprising consequence is that if a CT: ${ }^{*}$ formula is not equivalent to any CTI. formula, then it must have an infinite number of mutually inequivalent finite models. To see that this result is true, we first observe that since $\mathrm{CIL}^{*}$ has the finite model property, it must be the case that if two CTL" formulas have the same finite models, they must have the same infinite models as well. Otherwise. if $f_{1}$ had an infinite model $M$ that was not a model of $f_{2}, f_{1} \wedge \neg f_{2}$ would have an infinite model, but no finite models, contradicting the finite model property of CTL" [5]. Therefore, we can characterize a CTL" formula by the set of finite models in which it is satisfied. If a CTI. formula is satisficd by only a finite number of equivalence classes of finite models, then the formula is cquivalent to the disjuction of the CTL formulas that characterize the individual equivalence classes.

There are a number of directions for further research. First, from our construction, it appears that the characteristic formula of a Kripke structure might be quite large. It would be nice to have a lower bound on the size of this formula in terms of the size of the Kripke structure. Also, we conjecture that the $O\left(|S|^{6}\right)$ algorithm in Section 5 can be improved significantly. Finally, it would be interesting to see which of our results carry over to Kripke structures with fairness constraints, i.e. Büchi automata.

\section{References}

1. M. Ben-Ari, A. Pneuli, Z. Manna. "The Temporal Logic of Branching Time". Acta Informatica 20 (1983), 207-226.

2. E.M. Clarke, E.A. Emerson. Design and Synthesis of Synchronization Skelctons using Branching Time Temporal Logic. Proceedings of the Workshop on Logic of Programs, Yorkiown-Heights, NY, Lecture Notes in Computer Science \#131, 1981.

3. E.M. Clarke, E.A. Emerson, A.P. Sistla. Automatic Verification of Finite-State Concurrent Systems using Temporal Logic Specifications: A Practical Approach. Tenth ACM Symposium on Principles of Programming Languages, Austin, Texas, 1983, pp. 117-126.

4. E.A. Emerson, J.Y. Halpern. "Sometimes" and "Not Never" Revisited: On Branching versus Linear Time Temporal Logic. Proceedings of the ACM Symposium on Principles of Programming Languages, Association for Computing Machinery, Austin, Texas, January, 1982. to appear in JACM.

5. E. A. Emerson and P. Sistla. Deciding Full Branching-time Logic. The Sixteenth Annual ACM Symposium on Theory of Computing, Association for Computing Machinery, Washington, D.C., May, 1984.

6. S. Graf and J. Sifakis. From Synchronization Tree Logic to Acceptance Model Logic. LNCS Vol. 193, Logics of Programs, 1985.

7. M. Hennessy and R. Milner. On Observing Nondeterminism and Concurrency. LNCS Vol. 85, 7th ICALP, 1980.

8. G.E. Hughes and M.J. Creswell. An Introduction to Modal Logic. Methuen and Co., 1977.

9. L. Lamport. "Sometimes" is Sometimes "Not Never". Seventh Annual ACM Symposium on Principles of Programming Languages, Association for Computing Machinery, Las Vegas, January, 1980, pp. 174-185.

10. L. Lamport. What Good is Temporal Logic? Proceedings of the International Federation for Information Processing, 1983, pp. 657-668. 
11. O. I. ichtenstein and $\Lambda$. Paucli. Checking that linite State Concurrent Programs Satisfy lheir Linear

Specification. Conference Record of the Tweth Annual $\Lambda$ CM Sympusium on Principles of Programming I anguages, New Orlcans, 1a., January, 1985.

12. R. Milncr, Leciure Notes in Computer Science. Volume 92: A Calculus of Communirating Systems. SpringerVerlag, 1979.

13. R. Mishra and E. Clarke. "Hicrarchical Vcrification of Asynchronous Circuits using Temporal Logic". Theorctical Computer Science 38 (1985), 269-291 .

14. Z. Manna, P. Wolpcr. "Synthesis of Communicating Processes from Temporall Logic Specifications". ACM Transactions on Programming Languages and Systems 6 (1984), 68-93.

15. A. Pnucli. Lincar and Branching Structures in the Scmantics and Lugics of Reactive Systems. Proceedings of the 12th ICALP, 1985.

16. A.P. Sistla, EM. Clarke, "Complexity of Propositional Linear Temporal Logics". Journal of the Association for Conputing Machinery 32, 3 (July 1985), 733-749.

17. M.Y. Vardi, P. Wolper. An automata-theoretic approach to automatic program verification. Logic In Computer Science, Cambrideg, Massachusetts, June, 1986.

18. P. Wolper. Specification and Synthesis of Communicating Processes Using an Extended Temporal Logic. Ninth Annual ACM Symposium on Principles of P'rogramming Languages, Association for Computing Machinery, Abuquerque, New Mexico, January, 1982, pp. 20-33. 